\title{
El enfoque sistémico de la innovación: ventaja competitiva de las regiones
}

\author{
CARLOS SCHEEL MAYENBERGER, Ph.D. ${ }^{1,2}$ \\ Profesor Titular Emérito, Tecnológico de Monterrey, \\ EGADE Business School, México. \\ cscheel@itesm.mx
}

\section{RESUMEN}

Las empresas, eje del desarrollo económico, requieren de condiciones industriales, sociales y ambientales especiales para poder desarrollar su actividad en forma óptima. Si no existen estas condiciones adecuadas de región, no hay un desarrollo sostenido. Esto es evidente en países emergentes en donde no existen los elementos, ni la voluntad política ni empresarial para aprovechar el impacto de la innovación en el desarrollo económico, ambiental y social de estas regiones. En este documento se propone un mecanismo para replantear el impacto de la innovación sobre una actividad económica empresarial, el cual depende de la estructura de su ecosistema político, social y ambiental y de la democratización de la iniciativa para generar un desarrollo holístico regional de beneficio para todos.

Palabras clave. Innovación sistémica; ecosistemas regionales; desarrollo holístico regional; ventaja competitiva de las regiones; ciudades innovadoras.

Clasificación JEL: M14; M54

@

1 1. Este documento fue presentado como ponencia en Sesión Plenaria por un Conferencista Principal, en el marco del “II Simposio Iberoamericano de Estudios Gerenciales: Una mirada interdisciplinar a la innovación”, organizado por la revista académica Estudios Gerenciales bajo la dirección de la Facultad de Ciencias Administrativas y Económicas de la Universidad Icesi; el evento tuvo lugar los días 12, 13 y 14 de octubre de 2011, en la ciudad de Cali (Colombia). 2 Esta investigación es el resultado de un gran número de investigadores y estudiantes de posgrado, pertenecientes a la Cátedra de Investigación “Creación de Riqueza por Innovación y Tecnología” fondeada por la EGADE Business School del Tecnológico de Monterrey, Monterrey México, aquienes el autor desea agradecer sus contribuciones. 


\section{A systemic approach to innovation: a regional competitive advantage}

Companies, which are the drivers of economic development, should operate under special industrial, social, and environmental conditions that enable them to carry out their business activities in an optimal manner. If these proper conditions are not in place in a region, there can be no sustainable development. This is evident in emerging countries where there are no factors, or business or political will to take advantage of the impact of innovation on the economic, social, and environmental development of these regions. This document proposes a mechanism to rethink the impact of innovation on economic business activity that depends on the structure of not only its political, social, and environmental ecosystem, but also of the democratization of these kinds of initiatives in order to generate holistic regional development for all.

Keywords. Systemic innovation; regional ecosystems; holistic regional development; regional competitive advantage; innovative cities.

\section{es}

\section{O enfoque sistêmico da inovação, vantagem competitiva das regiões}

As empresas, eixos do desenvolvimento econômico, necessitam de condições industriais, sociais e ambientais especiais a fim de poderem desenvolver sua atividade de forma ótima. Se não existem essas condições de região adequadas, não há um desenvolvimento sustentado. Isto é evidente em países emergentes onde não existem os elementos, nem a vontade política ou empresarial, para aproveitar o impacto da inovação no desenvolvimento econômico, ambiental e social destas regiões. Este trabalho propõe um mecanismo para reconfigurar o impacto da inovação sobre uma atividade econômica empresarial, o qual depende da estrutura de seu ecossistema político, social e ambiental e da democratização da iniciativa, para gerar um desenvolvimento holístico regional benéfico para todos.

Palavras-chave. Inovação sistêmica; ecossistemas regionais; desenvolvimento holístico regional; vantagem competitiva das regiões; cidades inovadoras. 
La prosperidad crece en función de los espacios creativos...

de las unidades económicas, de la infraestructura, de las vías de comunicación, la movilidad...

Pero crece principalmente por la capacidad que tiene la ciudad... para aceptar nuevas ideas, a gente de otros lugares...para crear un nuevo espacio de ideas

interculturales y diversas...para innovar...junto a la tolerancia, al talento y a la tecnología...

(Adaptado de Florida, 2010)

\section{Introducción}

Las empresas, eje del desarrollo económico de los países de occidente, requieren de condiciones industriales, sociales y ambientales especiales para poder desarrollar su actividad en forma óptima. Si no existen las condiciones de región adecuadas, no hay un desarrollo sostenido. Esto es evidente en países emergentes, en donde no existen los elementos, la voluntad política ni empresarial para aprovechar en forma apropiada el impacto de la innovación en el desarrollo económico y social de estas regiones. Es por esto que, si se quiere hablar de innovación y de su impacto sobre el desarrollo regional, se debe incluir la compleja dependencia de la innovación con la estructura política y social de las regiones en donde se quiere implementar.

Esto se ha experimentado en innumerables intentos por apoyar a empresas que emergen y que se han constituido siguiendo todos los estándares de mejores prácticas mundiales organizacionales y administrativas, pero que se encuentran inmersas en un modelo macroeconómico de país totalmente diferente, y hasta hostil, de su estructura organizacional, operativa o de su propia estrategia de negocios.

Por ejemplo, es imposible que surjan emprendimientos exitosos en un régimen puramente socialista (exceptuando quizás el modelo autocrático chino que abordó un enfoque hibrido a conveniencia), en donde la democratización de las estructuras económico-políticas, requisito necesario para que las estrategias de innovación tengan éxito, han impedido la existencia de cualquier iniciativa -de emprendimientos o emprendedores-, que se desarrolle bajo este contexto. Lo mismo ocurre con los modelos de empresas sociales que tratan de operar en un entorno capitalista totalitario que solo persiga el lucro económico, minimizando cualquier objetivo social o ambiental que no genere un razonable retorno económico a la inversión.

Por lo tanto, gran parte del éxito de cualquier empresa que esté emergiendo de sectores noveles basados en tecnología o conocimiento, depende de las condicionesregión en donde se encuentren, del impacto que la industria pueda tener al agregar valor a sus prácticas empresariales y de sus vínculos académicos, financieros o gubernamentales.

Lo mismo ocurre con las industrias en donde están operando las empresas. ¿Qué hubiera ocurrido con la industria aeronáutica si el invento de los hermanos Wright se hubiera concentrado en unidades personales de propulsión aérea en lugar del transporte aéreo masivo?

De haber sido así, quizás los aeropuertos tendrían como única función la de los vuelos inter-continentales; el mismo automóvil quizás no existiría como lo es hoy, ni la industria metalmecánica, ni la de los motores, ni de los materiales, ni de la autónica, ni de las comunicaciones satelitales. El transporte masivo sería como los trasatlánticos de hoy y se tendrían unidades personales tipo motos aéreas o taxis colectivos pequeños, muy sofisticados para dar movilidad por vía aérea en forma autóctona.

Es por esto es que el impacto de la innovación sobre una actividad económica 
empresarial depende de la estructura de su ecosistema político, social y ambiental y de la democratización de la iniciativa para desarrollarse en el ecosistema regional como espacio de articulación de los sub-sistemas económico, socio-político y ambiental.

\section{Ciudades innovadoras base del ecosistema regional}

Sin los dos conceptos básicos anteriores, las iniciativas de innovación son poco probable que prosperen. Para empezar, es necesario analizar cómo están conformados los espacios del ecosistema regional que propician las cadenas o clúster de innovación, y las actividades de emprendimiento que los países industrializados han utilizado para posicionarlos notablemente en arenas globales.

El primer cuestionamiento clave es cuáles deben de ser las características de una región para que ésta pueda articular exitosamente a las actividades de la cadena de innovación de un determinado sector industrial, en una región geográfica determinada, bajo unas condiciones regionales hostiles y recursos muchas veces limitados de sus actividades empresariales y de la escasa calidad de vida de sus habitantes.

Un segundo cuestionamiento es en qué consiste y para qué sirve el operar como una ciudad innovadora, qué características tiene y como éstas ciudades posicionan a sus empresas, sus organizaciones y a sus empresarios a ser exitosos en arenas mundiales, así como aprovechar oportunidades excepcionales que solo las ciudades innovadoras pueden atacar para el bien de sus ciudadanos.

Finalmente, el último cuestionamiento es con qué capacidades y recursos se logran estos posicionamientos. Aquí se encuentra el talón de Aquiles de los países emergentes, dadas la hostilidad de las condiciones regionales (regulaciones, monopolios, sindicatos, gasto público, etc.), la escasez de recursos especializados, las deficientes infraestructuras físicas, las ineficaces tecnologías habilitadoras, la poca creatividad y la pobre asociatividad requeridas para ensamblar los clúster de innovación, requisito indispensable para competir y convivir industrialmente en la época actual. De lo anterior surgen algunos interrogantes adicionales, como son:

- ¿Qué son las ciudades innovadoras?

- ¿Qué tienen en común ciudades como Barcelona (España), Austin (Estados Unidos), Bangalore (India), Curitiba (Brasil), Dublín (Irlanda), Manchester (Inglaterra) o Metz (Francia)? (Scheel, 2011)

La mayoría de ellas ha implementado un Plan de Estado que, en forma disruptiva, ha logrado dar un gran salto a un éxito espectacular, sufriendo transformaciones profundas. De poseer un perfil bajo de acuerdo a los estándares mundiales, se han convertido en pocos años en ciudades notables, con actividades dinámicas vibrantes, globalmente reconocidas, pero no solo en el nivel económico, sino también en su comportamiento social y ecológico. Estas ciudades han roto con los paradigmas convencionales y han utilizado prácticas innovadoras para crear excelentes resultados que las han posicionado en los foros de clase mundial.

Este enfoque se basa en evidencia teórica y empírica que apoya la idea de que los individuos viven y trabajan en una economía basada en el conocimiento ${ }^{3}$ (Hospers, 2003) en la cual el conocimiento, la tecnología y la innovación están moldeando continuamente el sistema económico y el desarrollo de las regiones (Adler, 2001; Cooke \& Leydesdorff,

3 Hospers (2003) argumenta que existen algunos ingredientes que de manera conjunta pueden incrementar las posibilidades de una ciudad creativa, y en consecuencia, pueden contribuir a una economía de conocimiento urbana. En general estos factores son: (1) concentración, (2) diversidad e (3) inestabilidad. 
2006; Hospers, 2003; Kim \& Mauborgne, 1999; Luque, 2001; Powell \& Snellman, 2004).

En la actualidad, una innovación se desarrolla de una manera mucho más rápida que en el pasado (Cooke, 2002). En este sentido, el desarrollo del conocimiento está siendo visto como un fundamento del desarrollo económico y del desarrollo de la ciudades actuales (Cheng, Chong, Chen, Eldomiaty \& Millar, 2004) ya que juega un papel crítico en la creación de riqueza regional; pero hay que tener las condiciones adecuadas para aprovecharlo.

Por lo tanto, varios factores deben de incluirse para completar el kit de herramientas de los mecanismos de innovación requeridos para aprovechar las oportunidades que se presentan cuando la ciudad está preparada en innovación (i-readiness). Uno de ellos es que la innovación proviene de visionarios y campeones, individuos que se reúnen y difunden soluciones a las necesidades de la comunidad (Florida \& Gates, 2006; Florida, Mellander \& Stolarick, 2008) y que, bajo un ambiente adecuado, pueden capitalizar excepcionalmente esas iniciativas.

Con estas ideas en mente, se puede integrar estos conceptos en un término general de ciudades innovadoras (innovacities). Desde esta perspectiva una ciudad innovadora puede ser definida como un espacio geográfico con condiciones regionales especiales en el que la mayoría de los ciudadanos, empresarios (industriales, científicos, tecnólogos, docentes, políticos, etc.), con sus capacidades, sus interrelaciones y sus recursos regionales (normas, reglamentos, reformas, leyes, capital de riesgo, tecnologías, empresas, etc.), están constantemente en búsqueda de oportunidades de alto valor, y con creatividad ${ }^{4} \mathrm{y}$ comunidades auto-organizadas, ${ }^{5}$ pueden

\footnotetext{
4 Ver Florida (2010).

5 Este concepto proviene del enfoque teórico de la ciber-

lograr beneficios extraordinarios para todos sus sub-sistemas interrelacionados (social, económico y ambiental), creando ciudades con un desarrollo holístico sostenible único.

En un estudio llevado a cabo del 2007 al 2010 (Scheel, 2011) que se centró en un pequeño grupo de ciudades que realizaron transiciones tremendas en periodos específicos de tiempo, se muestra que las ciudades que han cambiado el paradigma tradicional de crecimiento constante hacia uno de avances extraordinarios, están actualmente muy bien posicionadas en el ámbito mundial. El estudio tenía dos objetivos importantes: identificar los puntos de inflexión donde las ciudades hicieron las grandes transformaciones en términos de posicionamiento de clase mundial y, posteriormente, identificar los mecanismos que permitieron que se produjeran las transiciones y transformaciones que originaron cambios a gran escala y que crearon espacios interdependientes en donde los ciudadanos, las comunidades sociales, las empresas y sus entornos locales naturales articularan un desarrollo armonioso y equilibrado.

Las ocho ciudades seleccionadas fueron capaces de romper con los paradigmas convencionales de crecimiento y ahora son excepcionales jugadores de clase mundial en el ámbito global; estas son: Auckland (Nueva Zelanda), Austin (Estados Unidos), Bangalore (India), Barcelona (España), $\mathrm{Cu}-$ ritiba (Brasil), Medellín (Colombia), Metz (Francia) y Stavanger (Noruega). En el estudio se observó que algunas de las ciudades habían hecho un rompimiento: de tener un crecimiento sostenido durante décadas, hasta niveles excepcionales de desarrollo económico y social de estándares mundiales, como resultado de la decisión de individuos y de la ejecución de mecanismos atípicos, para la creación de condiciones especiales nética organizacional y sistemas de control de Beer (1985). 
regionales y de la presencia de éxitos espectaculares. Estos impulsores incluyeron infraestructuras inteligentes, políticas sociales especiales, políticas tecnológicas, factores macro-económicos, políticas educativas rompientes, planes incluyentes de investigación científica, tecnología e innovación. Pero sobre todo, estas ciudades tuvieron una conciencia holística (en circunstancias y tiempos) de sus líderes ejecutores.

Combinando estos factores facilitadores, estas ciudades han producido polos regionales altamente atractivos, economías vibrantes, crecimiento social equitativo y, la mayoría de ellas, han articulado procesos sostenibles inclusivos con la toma de decisiones ciudadanas diseñadas para preservar el desarrollo de futuras generaciones.

El Gráfico 1 resume los comportamientos excepcionales, producto de los mecanismos que las ciudades han utilizado para lograr características únicas desarrolladas en periodos relativamente cortos de tiempo. Ellas han tenido: (1) desarrollo holístico sostenible; (2) atractores regionales (atractores de talento, cultura, socios industriales e inversión extranjera directa); (3) excelente calidad de vida; (4) fuertes sectores industriales competitivos; (5) reconocimiento mundial (branding); (6) programas de protección del medio ambiente y desarrollo sustentable de actividades de transformación urbana; (7) mercados regionales emergentes bien manejados; (8) estándares excelentes de planeación urbana; (9) gran número de empresas nuevas o de alto valor; (10) activos territoriales envidiables; $\mathrm{y}$ (11) programas de emprendimiento cívico y social notables y de gran impacto para toda la ciudadanía.

Estas ciudades han roto con los paradigmas convencionales de crecimiento a través de grandes innovaciones atípicas y han tenido desempeños de clase mundial que definen lo que se ha descrito como una innovacity (Scheel, 2011).

Gráfico 1. Desarrollo de la ciudad en términos de innovaciones revolucionarias

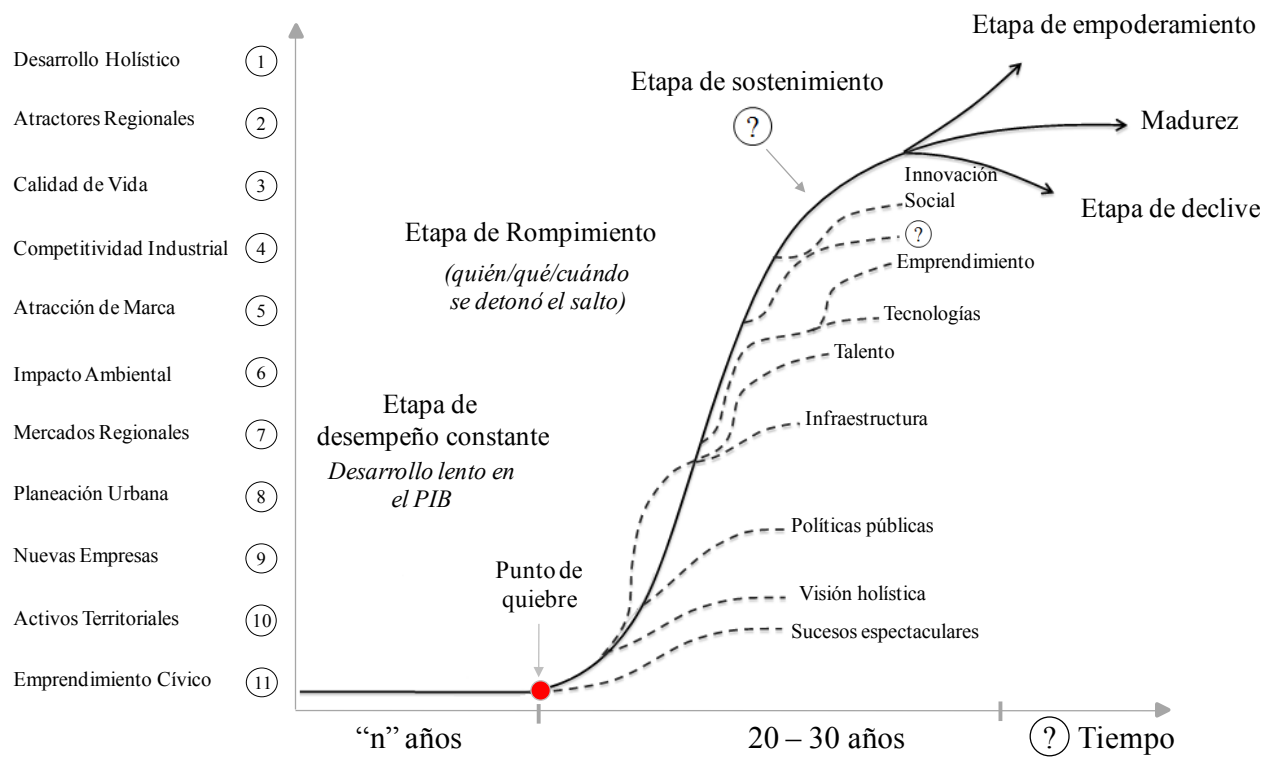

Fuente: Adaptado y traducido por los autores del documento de Scheel (2011, p. 381). 
A partir de estos casos, se ha observado que los procesos de innovación se han convertido en un pivote para el desarrollo humano, para la evolución social, la prosperidad económica y para la recuperación (resilencia) ambiental; cuando éstos se han diseñado desde una perspectiva sistémica y como el proceso central que articula las estructuras de innovación empresarial/ regional/nacional, las cuales pueden ser replicadas por otras regiones alrededor del mundo.

Una vez descrito el ecosistema regional, con sus características propias, cabe la pregunta ¿cómo se miden y cuáles han sido los impulsores que han utilizado estas ciudades para posicionarse globalmente y como apoyan en forma directa a las cadenas de innovación? Se puede concluir que para que la innovación genere resultados de estándares mundiales, requiere de un ecosistema en donde todos los jugadores estén holísticamente relacionados y con un mismo fin, el de crear una riqueza regional sustentable.

2 La innovación bajo un enfoque sistémico

De acuerdo con algunos autores (Senge, Smith, Kruschwitz, Laur \& Schley, 2008) son tres las capacidades que deben estar presentes en las organizaciones que aprenden (organizaciones aprendientes). Estas características son: (1) tener la capacidad de ver a los sistemas más grandes; (2) colaborar entre fronteras y (3) visualizar los futuros deseados.

Para que la innovación tenga éxito debe de estar notablemente conectada con los factores externos a donde se desarrolla. En este sentido, debe democratizarse y tener una gobernanza adecuada para poder articular a todos los agentes impulsores de la innovación con las condiciones industriales y de región hacia el mismo fin de creación de riqueza. Con las siguientes paradojas se aclaran estos conceptos:

- De amaestrador de perros en Jalisco a estrella de televisión en California.

- De emprendedor frustrado y fracasado en Monterrey a empresario de empresa de alta tecnología en el parque tecnológico de Austin.

- De programador frustrado en Argentina a empresario de software en Boston.

El éxito del emprendimiento depende primordialmente de los efectos de la ciudad sobre la cadena de innovación.

\section{La innovación forma parte de un fenómeno} social-geográfico

La innovación es un arma clave y competitiva en la era de la globalización (Cooke, 2002) y puede ser analizada desde diferentes perspectivas. A largo plazo, la innovación es lo que mantiene a las ciudades vibrantes y relevantes con gran apertura y fluidez de talento (Florida, 2010). El caso más destacado de Silicon Valley (Munroe \& Westwind, 2008) presenta resultados interesantes sobre la ecología de la innovación de una de las regiones basados en la tecnología y de mayor éxito en el mundo.

Por otro lado, algunos autores definen la innovación de productos y procesos así como la innovación estratégica desde la perspectiva microeconómica, y otros añaden la innovación social y política como un componente importante propicio para llegar a ser comunidades innovadoras.

Sin embargo, los procesos de innovación (industrial/pública/social/ciudadana, etc.) y las estructuras sistémicas, parecen ser los detonadores comunes para el avance realizado por las ciudades seleccionadas. Estos han sido capaces de ensamblar sistemas regionales de innovación sólidos (RIS) (Asheim \& Gertler, 2005; Cooke, 2001, 2002; 
Cooke, Urange \& Extebarria, 1997) que han creado una nueva perspectiva del territorio, una nueva geografía de trabajo y de vida, como lo ha descrito Florida (2010) con gran talento en varias de sus obras. Se trata de ensamblar espacios a través de la combinación de fuerzas políticas, culturales y económicas, todas involucradas en la creación, difusión y uso del conocimiento (Carlsson, Jacobsson, Holménb \& Rickne, 2002), así como la atracción de trabajadores y de las actividades intensivas en conocimiento (Hospers, 2003).

En estos espacios de innovación se va creando una coercividad cada vez mas fuerte entre ideas, individuos, redes de individuos, redes de comunidades colaborativas sociales y ecosistemas industriales con metas comunes de ganar-ganar para todos; es un hilo conductor desde el individuosociedad-industria-medioambiente; es una forma de democratización de las ideas hasta llegar a impactar a más gente, a más entornos, como lo ha hecho, por ejemplo, Barcelona en su plan integrado de innovación inclusiva.

Es realmente simple pasar de buenas ideas a mucho más impactantes ideas, como por ejemplo, de la bombilla eléctrica a un sistema de electrificación urbana. Siguiendo este ejemplo, se puede comentar que la electrificación ha tenido un enorme impacto en el desarrollo industrial de la humanidad. Este es un buen caso de cómo transferir la innovación del producto (bulbo) a la innovación del sistema industrial (sistema de electrificación). Lo mismo ocurrió con el paso del iPod al iTunes y a la creciente industria del entretenimiento de la i-música, a la $i$-cloud, y de paquetes administrativos a aplicaciones individuales a software accesado colectivamente de la $i$-cloud.

En esto consiste el concepto de innovación sistémica: el cómo transferir las ideas fundamento de una innovación de pro- ducto/servicio/proceso, hacia un impacto ambiental y democrático social, que sea económicamente viable y generador de riqueza sustentable y sostenible en el largo plazo.

Lo anterior quiere decir que, para competir en forma innovadora en un contexto globalizado, complejo y totalmente conectado, se requiere no solo de tener excelentes ideas o competencias medulares (core competencies), o una gran diferenciación de productos o servicios, o tener una red de clientes bien atrapados, o un nicho especializado de explotación. Actualmente, es necesario que además de todo lo anterior, la empresa, el emprendedor o la institución, estén insertados en un ecosistema industrial que, en forma sistémica e integrada, puedan fortalecer sus interrelaciones industriales, sociales y ambientales, porque hoy compiten regiones contra regiones con factores regionales que impactan a todos los habitantes con:

- Alta calidad de vida

- Reconocimiento mundial (branding) y de gran prestigio

- Atractores efectivos de talentos, cultura, socios industriales e inversión extranjera directa

- Conciencia holística efectiva de los líderes

(visión de largo plazo)

- Fuertes sectores industriales competitivos

- Gran número de nuevas empresas gene-

radoras de alto valor

- Planeación urbana armoniosa

- Programas de concientización de protección ambiental y desarrollo urbano sustentable

- Programas empresariales cívicos y sociales (para aquellos en la base de la pirámide)

- Mercados regionales crecientes de amplia cobertura

- Activos territoriales privilegiados (con la ubicación y los recursos naturales) 
- Cadenas completas de innovación (de ideas a empresas, de empresas a polos de desarrollo)

- Eventos espectaculares que ocurrieron en la región

Dado lo anterior, los países emergentes deben de eliminar su estado de determinismo histórico de sobrevivencia austera, aletargada e inerte, para construir sobre una dinámica de innovación sistémica. Esta transferencia es complicada, costosa y tardía, pues requiere rediseñar la burocracia gubernamental y la privada, transparentar las cuentas y acciones públicas, y ejecutar el estado de derecho, además de cambiar la actitud cultural de escases histórica por la de abundancia, y por el uso adecuado de la innovación tecnológica.

En esto consiste el concepto de innovación sistémica, un efecto democrático e incluyente que impulsa no solo a competir por mercados, sino que fuerza a las regiones a encontrar nuevas formas de crear riqueza; holísticamente hablando, requieren ser más atractivas, ecológicamente más efectivas, menos destructivas del ambiente, tener estándares de mejor calidad de vida, ser socialmente más equitativas y, finalmente, ser económicamente más independientes de las grandes transaccionales al entrar en ciclos de rendimientos crecientes de mayor valor (esto es, apostarle a sectores industriales de nicho emergentes de alto valor y diferenciación, en donde la tecnología tiene un role crucial, en áreas modernas de nanotecnología, biotecnología, nuevos materiales, etc.).

Siendo realistas, en los países emergentes esto resulta algo utópico en el presente si se siguen empleando las mismas reglas de política pública y empresarial. Pero si se innova en las estructuras, esto es posible en el largo plazo, lo que le trae al emprendedor, al empresario y al político en turno, una problemática creciente y típica del dilema del innovador (que actualmente se ha transformado en trilemma): ¿cómo pasar de tener una organización eficiente a una altamente innovadora? (este sería el dilema); lo que actualmente se convierte en cómo ser eficiente (en las finanzas), innovador (en procesos, servicios o productos), y además, cómo mantener el entorno ambiental regional sano, y al mismo tiempo tener una economía viable? (trilemma)

Esto obliga a los jugadores de la región (a todos los stakeholders) a evolucionar hacia un enfoque sistémico de generación de valor en donde todos ganen (ciudadanos, sociedad, empresas, industrias y medio-ambiente) y en donde el ciclo de vida de productos (cadenas de valor de producción) se integre a la ecología industrial del medio ambiente regional.

Esta innovación en la creación de valor, enfocada a una generación de riqueza sustentable, se puede resumir en: cómo atacar otros ciclos de vida (residuos y basuras) de mayor valor; cómo insertar la empresa hermética y convencional en ciclos económicos abiertos, de rendimientos crecientes y moverlas a áreas de mayor valor económico o social; cómo generar otros modelos de negocio y economías de red; cómo articular clusters robustos (polos competitivos) de innovación y con una gobernancia y administración de la ubicación de efectivos sistemas regionales de innovación; todo ello para generar un desarrollo regional holístico en el que todos gananen.

Lo anterior implica administrar la ubicación de los recursos (estén donde mejor valor generen) y una gobernancia sistémica de participación democrática de todos los integrantes de la región (ABIIGs). Igualmente, implica cerrar el ciclo económico al insertar la empresa convencional y hermética en ciclos económicos de rendimientos crecientes y trasladarla a áreas de mayor valor, insertándola en valiosas economías de red. Esto 
permitirá gobernar, sincronizar y coordinar a todos los participantes a través de los sistemas regionales de innovación, ensamblando clusters robustos (polos competitivos), o sea, transformar el mar rojo en un océano azul de nuevas posibilidades, no solo en las cadenas de producción de productos, sino también en la cadena de los sistemas de ecología industrial, considerando, residuos, basura y energía en el mismo ecosistema regional.

\section{La gobernancia y los sistemas regionales de innovación}

A través de este documento se ha ubicado la necesidad de ensamblar un ecosistema regional adecuado para promover la democratización de innovación para que esta tenga una cobertura amplia y dinámica. Pero, ¿cómo se administran y se gobiernan los recursos para llegar a tener un polo generador de riqueza y que, simultáneamente, esté alineado con la agenda de competitividad de la región?

Para ejercer esta actividad se requiere el concurso de varios actores, elementos constitutivos de los sistemas regionales de innovación (SRI) que articulen a todos los ABIIGS (academia, banca, industrias relacionadas, innovación e infraestructura, gobierno y sociedad), con el objetivo de:

- Gestionar la tecnología, la innovación y el conocimiento

- Gestionar la localización optima de los mejores recursos

- Gestionar las cadenas de innovación a través de:

- Investigación, generación de nuevas ideas y conceptos.

- Desarrollo de proyectos de innovación.

- Desarrollo de tecnologías y desarrollo de prototipos.

- Transferencia de tecnología a producto.

- Identificación y desarrollo de aplicaciones.

- Generación y expansión de mercados.
- Generación de nuevos productos, procesos y modelos de negocio.

- Generación de nuevas empresas.

- Aceleración de empresas.

- Ensamble de polos de competitividad e innovación.

- Gobernabilidad de la cadena de innovación: generación de acciones, políticas y estrategias para la generación de riqueza sustentable en la región.

De esta forma, se logrará una sinergia regional industrial y empresarial en los tres niveles de impacto de la innovación (empresas, industrias y regiones), en donde se obtengan regiones con:

- Una mejor identidad y branding de la región ante inversionistas internacionales,

- Alto valor agregado de la región sobre el PIB (nacional o regional),

- Valor diferencial y único en la región,

- Empleos mejor remunerados de mayor valor económico agregado (EVA),

- Más altos impuestos provenientes de actividades con un alto EVA,

- Alto grado de especialización de la región ante el país y el mundo,

- Mejor posición en los indicadores de calidad de vida de los ciudadanos,

- Exportaciones con mayor valor agregado

(alta y mediana tecnología involucradas),

- Mayor volumen de empresas nuevas de

base tecnológica,

- Sociedades financieras establecidas en la región para apoyar el capital de riesgo emprendimiento (NVC),

- Mejor posición en el Indicador Global de Progreso (GPI),

- Regiónes con mecanismos efectivos de repartición del capital económico en obras y programas de desarrollo social y ambiental, transparentes y de gran impacto,

- Alta influencia de las empresas en los proyectos de investigación (con un alto porcenta- 
je de inversión en investigación por empresas locales) de alto impacto y de largo tiempo, - Regiones con una visión sistémica de sus líderes (visionarios, campeones, creyentes, seguidores) con un plan de desarrollo holístico (plan incluyendo: ecosistema social, económico, ambiental, inclusivo y con valores tolerantes (diseñado acorde a los principios humanos y un estricto apego al estado de derecho),

- Regiones centradas en las actividades académicas de una gran universidad o centros de investigación, que le brinde ese toque de especialización y unicidad,

- Universidades con altos presupuestos en investigación y programas de posgrado especializados,

- Y con todo esto, una gran y bien estructurada cultura de emprendedor que abarque a toda la red de jugadores industriales, sociales, académicos, políticos y ecológicos dela región.

En resumen, los sistemas regionales de innovación logran administrar la ubicación de los recursos y la gobernancia de la trilogía (empresa-industria-región) en donde los recursos, las condiciones y las relaciones, se deben redefinir hacia la formación de ecosistemas regionales basadas en la relación tecno-económica-social, en donde todos ganan y mejoran la calidad de vida para los habitantes de la región.

\section{Conclusiones}

Las ciudades que están preparadas para articular ecosistemas regionales son capaces de aprovechar oportunidades mundiales que solo se presentan cuando hay ciertas condiciones muy especiales de gobernancia de los sistemas de innovación regionales, de la industria-región y de las competencias medulares (core competencies) de las empresas que forman parte del tejido industrial de la región.

En las ciudades en las que la innovación se convirtió en el emblema del ciclo de creci- miento y competitividad, se observa que han tenido un líder visionario; han tenido un desarrollo holístico; todas se han desarrollado como clúster alrededor de una universidad prestigiosa o centro de investigación; han tenido una política pública alineada totalmente al desarrollo tecno-industrial, social y ambiental de la región; han creado una sinergia para que la tecnología sea un eje muy importante de desarrollo; han tenido un efectivo y abarcante espíritu emprendedor; y finalmente, han podido administrar y gobernar sus recursos mediante un sistema regional de innovación (SRI) articulado con todos los jugadores pertenecientes a la sinergia entre la academia, la banca, la innovación e infraestructura inteligente, las industrias relacionadas, el gobierno y con/para el ciudadano y la sociedad (ABIIGS).

Esto es posible porque, una vez las regiones se encuentran en este ciclo de rendimientos crecientes de valor, es relativamente sencilla la creación de riqueza en una forma sustentable y de beneficio para todos.

El mensaje es claro, hoy la creación de riqueza social, económica y ambiental requiere de la articulación sistémica de todos los miembros de las regiones -que son quienes compiten-, porque a la medida que éstas son exitosas, lo son sus industrias y sus empresas, y por ende sus sociedades y ciudadanos.

Para alcanzar las grandes oportunidades que están abiertas para todos en este mundo plano, complejo y totalmente interconectado, hay que ser creativos, innovadores y actuar según una ética sustentable de beneficio para todos. La naturaleza no crea elites ni feudos, cada miembro tiene sus capacidades y su entorno para aprovecharla, sin excesos ni desechos, y al ser parte de la naturaleza, asíse debe de actuar, porque gana el que mejor se adapte a las circunstancias y quiera compartir sus utilidades de la mejor manera posible. 
La clave está en la creación de estos espacios de ecosistemas regionales para que, en forma articulada y sistémica, todos puedan co-existir en forma democrática. De esta forma, los tres sub-sistemas del ecosistema natural de la biosfera serán socialmente equitativos y responsables; ambientalmente reversibles, recuperables y durables; y económicamente viables. En conclusión, innovar con una conciencia holística de todas las partes, en donde el crecimiento económico, desarrollo social y el impacto ecológico, converjan en un único concepto de desarrollo sistémico regional.

\section{Referencias bibliográficas}

Adler, P.S. (2001). Market hierarchy and trust: The knowledge economy and the future of capitalism. Organization Science, 12(2), 215-234.

Asheim, B.T. \& Gertler, M. (2005). The Geography of Innovation: Regional Innovation Systems. En J. Fagerberg, D.C. Mowery \& R.R. Nelson (Eds.), The Oxford Handbook of innovation (pp. 291-317). New York, NY: Oxford University Press.

Beer, S. (1985). Diagnosing the system for organizations. New York, NY: Wiley.

Carlsson, B., Jacobsson, S., Holménb, M. \& Rickne, A. (2002). Innovation systems: analytical and methodological issues. Research Policy, 31(2), 233-245.

Cheng, P., Chong, J., Chen, S., Eldomiaty, T. \& Millar, C. (2004). Knowledge repositories in knowledge cities: institutions, conventions and knowledge subnetworks. Journal of Knowledge Management, 8(5), 96-106.

Cooke, P. (2001). Regional innovation systems, clusters, and the knowledge economy. Industrial and Corporate Change, 10(4), 945-974.
Cooke, P. (2002). Knowledge Economies. Clusters, Learning and Cooperative Advantage. Londres/New York: Routledge.

Cooke, P. \& Leydesdorff, L. (2006). Regional development in the knowledge-based economy: the construction of advantages. Journal of Technology Transfer, 31(1), 5-15. Cooke, P., Urange, M.G. \& Extebarria, E. (1997). Regional innovation systems: institutional and organizational dimensions. Research Policy, 4(5), 475-493.

Florida, R. (2010). The Great Reset. New York, NY: Harper Collins Publishers.

Florida, R. \& Gates, G. (2004). Technology and Tolerance: The Importance of Diversity to high-technology Growth. En T.N. Clark (Ed.), The City as an Entertainment Machine (pp. 199-219). London: JAI.

Florida, R., Mellander, C. \& Stolarick, K. (2008). Inside the Black Box of Regional Development - Human Capital, the Creative Class and Tolerance. Journal of Economic Geography, 8(5), 615-649.

Hospers, G.-J. (2003). Creative cities: breeding places on the knowledge economy. Knowledge, Technology, and Policy, 16(3), 143-172.

Kim, W.C. \& Mauborgne, R. (1999). Creating the new market space. Harvard Business Review, 77(1), 83-93.

Luque, E. (2001). Whose knowledge economy? Social Epistemology, 15(3), 187-200.

Munroe, T. \& Westwind, M. (2008). Silicon Valley: The Ecology of Innovation. Malaga, España: Euromedia Comunicación.

Powell, W.W. \& Snellman, K. (2004). The knowledge economy. Annual Review of Sociology, 30, 199-220.

Scheel, C. (2011), Innovacities: in search of breakthrough innovations producing world-class performance. International Journal of Knowledge-based development. 2(4), 372-388. 
Senge, P., Smith, B., Kruschwitz, N., Laur, J. \& Schley, S. (2008). The necessary revolution: How Individuals and Organizations Are Working Together to Create a Sustainable World. New York, NY: Knopf Doubleday. 